\title{
ADJOINT SENSITIVITY ANALYSIS IN NUCLEAR REACTOR FUEL BEHAVIOR MODELING
}

\author{
Ronald A. CHRISTENSEN and Richard F. EILBERT \\ Entropy Limited, South Great Road, Lincoln, MA 01773, USA \\ Ronald A. ROHRER \\ University of Colorado, Department of Electrical Engineering, Computer Science, Colorado Springs, CO 80906, USA
}

Gary S. WAS

University of Michigan, Department of Nuclear Engineering, Ann Arbor, MI 48109, USA

Received 1 April 1981

\begin{abstract}
A computer program, SCODE, has been developed for calculating sensitivities for EPRI's SPEAR-ALPHA nuclear fuel performance code FCODE-ALPHA. Eleven critical parameters are assessed for the effects of their independent variations on 33 basic variables in the FCODE-ALPHA model. The enormous wealth of sensitivities that result, consisting of 363 quantities per axial node per time step, are calculated following FCODE-ALPHA computations on each time step. SCODE is based on adjoint sensitivity analysis, which is an analytic technique, obviating the need for numerical differentiation via repeated code runs at varied parameter values. Evaluation of sensitivities is reduced to a problem in linear algebra and is handled by standard matrix manipulations. Compared with the customary numerical differentiation approach, SCODE offers advantages of significant runtime reduction, exactitude of results, and on-line computation of sensitivities.
\end{abstract}

\section{Introduction}

An important aspect of problem solving using large, complex computer codes is developing the capability to determine how the output information depends on the input information. Knowledge of these dependencies serves a dual purpose. First, they act as a feedback mechanism by indicating just how sensitive the outcome information (output variable) is to a change in the input information. This aids in establishing operating constraints, or setting tolerance levels on the input parameters, and therefore the actual hardware of the system under study. Second, sensitivities of output variables with respect to input parameters or other independent variables can be used to speed up convergence of numerical solution procedures by providing accurate and up-to-date estimates of the values of variables that are defined by complicated or implicit functional relationships.

The fuel behavior code used in this analysis (FCODE-ALPHA, a component of EPRI's SPEAR-ALPHA) [1] constitutes a set of equations that, as a unit, describe the thermomechanical behavior of a fuel rod subject to a specific set of input conditions. A code run solves these equations in a predetermined sequence so as to yield the specified output information. Sensitivity analysis reveals how, at any time during the operation of the code, a variable changes with respect to a change in any input parameter. Since the variables are, in general, interrelated, all variations must be solved for simultaneously.

Sensitivities are obtained by performing variations about a known operating point, that is, the relevant quantities have been calculated up to the current time step. The same equations that are used to solve the original problem are cast in variational form to solve for the sensitivities. In the case where some of these 
equations appear in integral or differential form the variational problem is handled through adjoint sensitivity analysis [2,3]. Adjoint sensitivity has its roots in the calculus of variations of the Lagrange form [4]. This paper reports on the SCODE implementation [5-7] of adjoint sensitivity to the problem of nuclear reactor fuel behavior modeling. Other investigators have explored sensitivity analysis for various aspects of reactor technology $[8-10]$.

\section{Theory}

The models in FCODE-ALPHA consist of two types of equations:

(a) Differential equations:

$$
\dot{x}_{m}=f_{m}(\boldsymbol{x}, \boldsymbol{y}, \boldsymbol{p}, t) \quad m=1, M
$$

and

(b) Constraint equations:

$$
y_{n}=g_{n}(\boldsymbol{x}, \boldsymbol{y}, \boldsymbol{p}, t) \quad n=M+1, N .
$$

Here the dot notation refers to the complete derivative with respect to time $t$, which includes implicit dependencies through the variables $\boldsymbol{x}$ and $\boldsymbol{y}$ as well as any explicit time dependence. The parameter set $\boldsymbol{p}$, or $p_{k}(k=1, \ldots, K)$, represent any constants in the analytic model for which the sensitivity is sought. Eq. (1) may be rewritten as

$$
\dot{x}_{m}-f_{m}(\boldsymbol{x}, \boldsymbol{y}, \boldsymbol{p}, t)=0, \quad y_{n}-g_{n}(\boldsymbol{x}, \boldsymbol{y}, \boldsymbol{p}, t)=0 .
$$

A differential equation is one in which a variable is defined in terms of a relation given for its derivative. An example is the equivalent thermal clad creep strain equation.

$$
\dot{\epsilon}_{\mathrm{eq}, \mathrm{th}}=m C \sigma_{\mathrm{eq}}^{n / m_{\mathrm{eq}, \mathrm{h}}}(m-1) / m\left[\exp \left(\alpha \frac{T_{\mathrm{c}}-300}{T_{\mathrm{c}}+300}\right)\right]^{1 / m},
$$

where $\sigma_{\mathrm{eq}}$ is the current equivalent stress and $T_{\mathrm{c}}$ is the current clad average temperature, $m, n, C$ and $\alpha$ are constants.

A constraint equation is one in which no derivatives appear and the variable is expressed solely in terms of other variables. An example is the fuel surface temperature equation

$$
T_{\mathrm{F}}=T_{\mathrm{CI}}+\frac{q_{\mathrm{L}}}{2 \pi R_{\mathrm{F}} h_{\mathrm{g}}}
$$

where $T_{\mathrm{CI}}$ is inner clad temperature, $R_{\mathrm{F}}$ is fuel radius, and $h_{\mathrm{g}}$ is gap conductance. If all equations in the FCODE model were constraint equations, then the sensitivity coefficients could be found simply by writing the variations of each equation in matrix form and applying a standard matrix inversion routine.

For a system such as ours, involving also differential equations, the solution procedure is somewhat more involved. Suppose we want to find the sensitivity of a variable $X$, defined either by a differential or constraint equation, with respect to the selected set of parameters $p$. To aid our accomplishment of this, we introduce adjoint functions $\phi_{m}(t)$ and $\psi_{n}(t)$ conjugate to variables $x_{m}$ and $y_{n}$. Now we take variations of eq. (2), multiply them by $\phi_{m}(t)$ and $\psi_{n}(t)$ respectively, add them together and integrate over time. This gives (with summation implied on repeated indices)

$$
0=\int_{A}^{B}\left[\phi_{m}\left(\delta \dot{x}_{m}-\delta f_{m}\right)+\psi_{n}\left(\delta y_{n}-\delta g_{n}\right)\right] \mathrm{d} t .
$$

As will be seen, eq. (5) reduces to a particularly simple form if we require that the arbitrary functions $\phi_{m}$ 
and $\psi_{n}$ satisfy the adjoint relations

$$
\dot{\phi}_{m}=-\frac{\partial}{\partial x_{m}}(\boldsymbol{\phi} \cdot \boldsymbol{f}+\boldsymbol{\psi} \cdot \boldsymbol{g}), \quad \psi_{n}=\frac{\partial}{\partial y_{n}}(\boldsymbol{\phi} \cdot \boldsymbol{f}+\boldsymbol{\psi} \cdot \boldsymbol{g}) .
$$

Here $\phi(t)$ and $\psi(t)$ are regarded as functions of $t$ only so that the following identities hold

$$
0 \equiv \frac{\partial \phi_{m}}{\partial \boldsymbol{x}}=\frac{\partial \phi_{m}}{\partial \dot{\boldsymbol{x}}}=\frac{\partial \phi_{m}}{\partial \boldsymbol{y}}=\frac{\partial \phi_{m}}{\partial \boldsymbol{p}}, \quad 0 \equiv \frac{\partial \psi_{n}}{\partial \boldsymbol{x}}=\frac{\partial \psi_{n}}{\partial \dot{\boldsymbol{x}}}=\frac{\partial \psi_{n}}{\partial \boldsymbol{y}}=\frac{\partial \psi_{n}}{\partial \boldsymbol{p}}
$$

The first step toward evaluating eq. (5) is to expand $\delta \boldsymbol{f}$ and $\delta \boldsymbol{g}$ in terms of their dependencies. Time itself is not varied, but model parameters are varied and they induce variations in the solution set of the $x$ and $y$ variables. Thus,

$$
\delta f_{m}=\frac{\partial f_{m}}{\partial \boldsymbol{x}} \boldsymbol{\delta} \boldsymbol{x}+\frac{\partial f_{m}}{\partial \boldsymbol{y}} \boldsymbol{\delta} \boldsymbol{y}+\frac{\partial f_{m}}{\partial \boldsymbol{p}} \boldsymbol{\delta} \boldsymbol{p}, \quad \boldsymbol{\delta} g_{n}=\frac{\partial g_{n}}{\partial \boldsymbol{x}} \delta \boldsymbol{x}+\frac{\partial g_{n}}{\partial \boldsymbol{y}} \boldsymbol{\delta} \boldsymbol{y}+\frac{\partial g_{n}}{\partial \boldsymbol{p}} \boldsymbol{\delta} \boldsymbol{p}
$$

Next, the $\delta \dot{x}$ term in eq. (5) can be eliminated through an integration by parts

$$
\int_{A}^{B} \boldsymbol{\phi} \cdot \boldsymbol{\delta} \dot{\boldsymbol{x}} \mathrm{d} t=\int_{A}^{B}\left[\frac{\mathrm{d}}{\mathrm{d} t}(\boldsymbol{\phi} \cdot \boldsymbol{\delta} \boldsymbol{x})-\dot{\boldsymbol{\phi}} \cdot \delta \boldsymbol{x}\right] \mathrm{d} t
$$

Substituting eq. (8) for $\delta f_{m}$ and $\delta g_{n}$ in eq. (5) and using eq. (9) gives

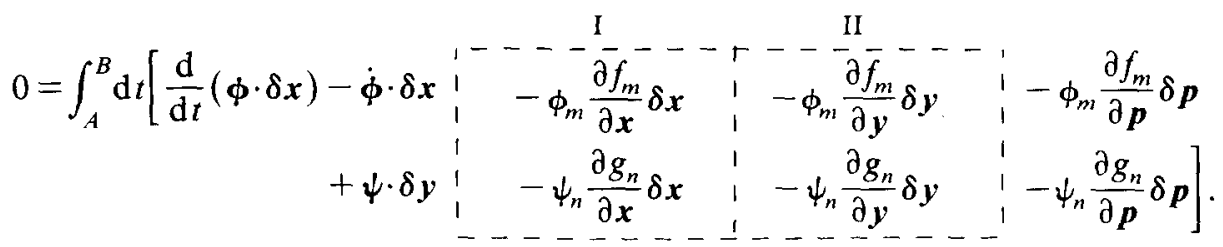

Because of the adjoint relations (6), the terms in box I sum to $\dot{\phi} \cdot \delta \boldsymbol{x}$ while those in box II sum to $-\boldsymbol{\psi} \cdot \boldsymbol{\delta} \boldsymbol{y}$. Eq. (10) now reduces to

$$
0=\int_{A}^{B} \mathrm{~d} t\left[\frac{\mathrm{d}}{\mathrm{d} t}(\boldsymbol{\phi} \cdot \delta \boldsymbol{x})+\boldsymbol{\delta} \boldsymbol{p} \cdot\left(-\boldsymbol{\phi}_{m} \frac{\partial f_{m}}{\partial \boldsymbol{p}}-\psi_{n} \frac{\partial g_{n}}{\partial \boldsymbol{p}}\right)\right]
$$

This complete differential term can be integrated, yielding

$$
\left.\boldsymbol{\phi} \cdot \boldsymbol{\delta} \boldsymbol{x}\right|_{A} ^{B}=\int_{A}^{B} \delta \boldsymbol{p} \cdot\left(\phi_{m} \frac{\partial f_{m}}{\partial \boldsymbol{p}}+\psi_{n} \frac{\partial g_{n}}{\partial \boldsymbol{p}}\right) \mathrm{d} t .
$$

Since $\phi$ and $\psi$ are by definition independent of $p$, we may take the partial differentiation outside the integral to obtain the variational relation

$$
\left.\boldsymbol{\phi}(t) \cdot \delta \boldsymbol{x}(t)\right|_{A} ^{B}=\delta \boldsymbol{p} \cdot \frac{\partial}{\partial \boldsymbol{p}} \int_{A}^{B}(\boldsymbol{\phi} \cdot \boldsymbol{f}+\psi \cdot \boldsymbol{g}) \mathrm{d} t .
$$

It may be shown that the eq. (13) is valid even if the parameter set is extended to include the limits of integration.

While eqs. (6) and (13) have an elegant form, they involve constructing a complete set of $N$ adjoint functions, where $N$ is the number of system equations. In principle, all the variables $y(t)$ can be expressed as functions of the $\boldsymbol{x}(t)$ and the system reduced from $N$ to $M$ equations. The adjoint functions $\psi(t)$ would then never enter the problem. In practice the elimination of variables $y$ may be extremely tedious.

In handling a large system of equations for sensitivity analysis, a workable approach is to choose a 'basic' set of variables consisting of all the $M$ differential equation variables $x(t)$ and a selection of $N-M$ key variables $y(t)$. Many 'auxiliary' variables must be explicitly eliminated from the system of equations in favor of $\{\boldsymbol{x}(t), \boldsymbol{y}(t)\}$. Eqs. (1) presuppose that such a reduction has already been effected. Adjoint functions are, of course, constructed only for the basic variables $\{x(t), y(t)\}$. 


\section{SCODE: a method for determining the sensitivities in FCODE-ALPHA}

A total of 33 equations is used to define a closed system of equations for sensitivities analysis of FCODE-ALPHA models. Two of these are differential equations ( $x$-type) and 31 are constraint equations

Table 1

SCODE basic variables. Creep variables, No. 1 and 2, enter in the FCODE-ALPHA model through differential equations. The other 31 variables enter in the form of constraint (i.e., algebraic) equations.

\begin{tabular}{|c|c|c|c|c|}
\hline No. & Variable & ID & Units & Defintion \\
\hline 1 & $\epsilon_{\theta, \mathrm{c}}^{*}$ & $\mathrm{EX}$ & $(-)$ & Renormalized circumferential clad crecp strain a \\
\hline 2 & $\epsilon_{\text {eq.th }}^{*}$ & EY & $(-)$ & Renormalized equivalent thermal clad creep strain ${ }^{h}$ \\
\hline 3 & $\sigma_{\theta}$ & ST & $(\mathrm{Pa})$ & Circumferential clad stress \\
\hline 4 & $\sigma_{z}$ & $S Z$ & $(\mathrm{~Pa})$ & Axial clad stress \\
\hline 5 & $\sigma_{e q}$ & $\mathrm{SE}$ & $(\mathrm{Pa})$ & Equivalent clad stress (Tresca) \\
\hline 6 & $R_{\mathrm{CO}}^{\prime}$ & RO & $(\mathrm{m})$ & Unrestraincd clad outer radius \\
\hline 7 & $R_{\mathrm{CI}}^{\prime}$ & RI & (m) & Unrestrained clad inner radius" \\
\hline 8 & $\epsilon_{\mathrm{el}}$ & EL & $(-)$ & Clad elastic circumferential strain \\
\hline 9 & $\underline{\epsilon_{g}}$ & $\mathrm{EG}$ & $(-)$ & Clad irradiation growth strain \\
\hline 10 & $\overline{\alpha_{c} T_{c}}$ & $\mathrm{AC}$ & $(-)$ & Clad thermal expansion strain \\
\hline 11 & $T_{\mathrm{CI}}$ & TI & $\left({ }^{\circ} \mathrm{C}\right)$ & Clad inside temperature \\
\hline 12 & $G_{\mathrm{Rel}}$ & GR & (mole) & Fission gas released since startup \\
\hline 13 & $F_{\mathrm{Re} e}$ & FR & $(-)$ & Fractional fission gas released since startup \\
\hline 14 & $p_{1}$ & PI & $(\mathrm{Pa})$ & Rod internal gas pressure \\
\hline 15 & $V_{\mathrm{T}}$ & VT & $\left(\mathrm{m}^{3} /{ }^{\circ} \mathrm{K}\right)$ & Total rod free-volume to temperature ratio \\
\hline 16 & $Z_{F, \text { hot }}$ & $Z F$ & $(\mathrm{~m})$ & Restrained crack width at fuel surface d.c \\
\hline 17 & bu & $\mathrm{BU}$ & $(\mathrm{MWd} / \mathrm{MTOx})$ & Burnup \\
\hline 18 & $\epsilon_{\mathrm{sw}}$ & ES & $(-)$ & Circumferential swelling strain \\
\hline 19 & $R_{\mathrm{F}, \text { cold }}^{*}$ & RS & (m) & Cold fuel radius after swelling ${ }^{f}$ \\
\hline 20 & $T_{\mathrm{F}}$ & $\mathrm{TF}$ & $\left({ }^{\circ} \mathrm{C}\right)$ & Fuel surface temperature \\
\hline 21 & $h_{g}$ & $\mathrm{HG}$ & $\left(\mathrm{W} / \mathrm{m}^{2 \circ} \mathrm{C}\right)$ & Fuel/clad gap conductance \\
\hline 22 & $k_{\mathrm{g}}^{\mathrm{g}}$ & $\mathrm{KG}$ & $\left(\mathrm{W} / \mathrm{m}^{\circ} \mathrm{C}\right)$ & Conductivity of gap gas mixture \\
\hline 23 & $T_{0}^{8}$ & $\mathrm{~T} 0$ & $\left({ }^{\circ} \mathrm{C}\right)$ & Fuel centerline temperature \\
\hline 24 & $T_{\mathrm{K}}$ & TK & $\left({ }^{\circ} \mathrm{C}\right)$ & Temperature at the fuel eracking radius 8 \\
\hline 25 & $R_{\mathrm{F}}^{\prime}$ & $\mathrm{RP}$ & $(\mathrm{m})$ & Unrestrained fuel radius ${ }^{\circ}$ \\
\hline 26 & $R_{F, c, h o t}^{\prime}$ & RH & (m) & Unrestrained fuel cracking radius c.g \\
\hline 27 & $R_{\mathrm{F}, \mathrm{c}, \mathrm{cold}}$ & $\mathrm{RC}$ & (m) & Cold fuel cracking radius ${ }^{i g}$ \\
\hline 28 & $t_{\mathrm{g}}^{\prime}$ & $\mathrm{TG}$ & $(\mathrm{m})$ & Unrestrained fuel/clad radial gap " \\
\hline 29 & $p_{\mathrm{c}}$ & $\mathrm{PC}$ & $(\mathrm{Pa})$ & Fucl/clad contact pressure \\
\hline 30 & $w_{\mathrm{F}}$ & WF & $\left(\mathrm{Pa}^{-1}\right)$ & Fuel clastic compliance \\
\hline 31 & $Z_{\mathrm{F}, \mathrm{c}, \text { hot }}^{\prime}$ & $\mathrm{ZP}$ & (m) & Unrestrained crack width at the cracking radius $c, c, g$ \\
\hline 32 & $R_{\mathrm{F}}$ & $\mathrm{RF}$ & (m) & Restrained fuel radius ${ }^{d}$ \\
\hline 33 & $R_{\mathrm{F}, \mathrm{c} \text {.hot }}$ & RK & (m) & Restrained fuel cracking radius ${ }^{d . f}$ \\
\hline
\end{tabular}

${ }^{a}$ Circumferential clad creep strain is renormalized to avoid an infinite primary creep rate at startup according to the transformation $\epsilon_{\theta, \mathrm{c}}^{*}=\epsilon_{\mathrm{eq}, \mathrm{th}}^{1 / m} \epsilon_{\theta, \mathrm{c}}$, where $\epsilon_{\theta, \mathrm{c}}$ is the clad circumferential creep strain, $\epsilon_{\mathrm{eq}, \mathrm{th}}$ is the equivalent thermal creep strain, and $m$ is the exponent in the time hardening rule $(m=0.3)$.

b Equivalent thermal ereep strain is renormaized to avoid an infinite primary creep rate at startup according to the transformation $\epsilon_{\text {eq, th }}^{*}=\epsilon_{\mathrm{eq}, \mathrm{th}}^{1 / m}$, where $\epsilon_{\mathrm{eq}, \mathrm{th}}$ and $m$ are defined in footnote a.

- Unrestrained dimensions include all effects except PCMI. In particular, negative unrestrained fuel/clad gap is allowed and signals the existence of contact pressure.

d Restrained dimensions account for all effects including restraint due to PCMI if contact pressure conditions exist.

c The crack width at a given fuel radius is the total crack distance existing on that circumference. Cracks are presumed to be radially oriented and to be produced via differential thermal expansion of the fucl.

f Cold dimensions refer to a hypothetical fuel state in which thermal contraction (to room temperature conditions) is assumed to have occurred.

g The cracking radius or crack tip is the outermost radius for which PCMI has forced the cracks closed within the fuel. 
Table 2

SCODE parameters. These 11 entities are inputs to the FCODE-ALPHA model. Sensitivities represent the variations in FCODEALPHA output variables induced by independent variations in these parameters

\begin{tabular}{rllll}
\hline No. & Variable & ID & Units & Definition \\
\hline 1 & $t_{\mathrm{F}}$ & TIME & $(\mathrm{hr})$ & Time (length of time since start-up) \\
2 & $Q_{\mathrm{L}}$ & QL & $(\mathrm{W} / \mathrm{m})$ & Linear heat generation rate on current time step \\
3 & $p_{\mathrm{O}}$ & PO & $(\mathrm{Pa})$ & Outer pressure on clad \\
4 & $p_{\text {I.fab }}$ & PIFAB & $(\mathrm{Pa})$ & Initial fill gas pressure \\
5 & $T_{\mathrm{CO}}$ & TCO & $\left({ }^{\circ} \mathrm{C}\right)$ & Temperature of clad outer radius \\
6 & $R_{\mathrm{CO}, \mathrm{fab}}$ & RCOFAB & $(\mathrm{m})$ & Fabricated outside clad radius \\
7 & $R_{\mathrm{CI}, \mathrm{fab}}$ & RCIFAB & $(\mathrm{m})$ & Fabricated inside clad radius \\
8 & $\alpha_{\mathrm{Q}}$ & QLT & $(-)$ & Scale factor on entire linear power history \\
9 & bu & BDEN & $(\mathrm{MWd} / \mathrm{MTOx})$ & Fuel burnup induced densification rate \\
10 & $P_{\text {fab }}$ & PFAB & $(-)$ & Fabricated fuel porosity \\
11 & $R_{\mathrm{F}, \mathrm{fab}}$ & RFFAB & $(\mathrm{m})$ & Fabricated fuel radius \\
\hline
\end{tabular}

( $y$-type). In the sensitivities solution procedure, the first two of these 33 variables are the differential variables and are treated specially. These variables, $\epsilon_{\theta, \mathrm{c}}^{*}$ and $\epsilon_{\mathrm{eq}, \mathrm{th}}^{*}$, relate to cladding creep which is defined by differential rather than algebraic equations. The remaining 31 variables enter in the form of constraint equations. The listing of these 33 variables is given in table 1 . Table 2 lists eleven input parameters selected for examination of effects of their variation. Examples of some auxiliary variables eliminated in favor of the basic set are presented in table 3.

The basic idea for handling sensitivities by SCODE is to convert the equations in the theory section to forms that are explicitly linear and that may be solved by standard matrix algebra. We employ a notation where parentheses are used to denote a vector and brackets denote a matrix. For clarity the vector or matrix dimensions are explicitly denoted using subscripts. A part of the vector or matrix is indicated by the use of a colon separating subscripts for the desired range. Components are indicated by the same letter but without parentheses or brackets. Then let

$(\delta X)_{N}=$ column vector of the $N$ dependent variations; $\delta x_{1}, \delta x_{2}, \delta y_{1}, \ldots, \delta y_{31}$. This vector subsumes both $x$-type and $y$-type variables, $(N=33)$.

$\left(\delta X^{\prime}\right)_{N}=$ column vector of the $N$ dependent variations with $\delta \dot{x}_{1}$ and $\delta \dot{x}_{2}$ replacing $\delta x_{1}$ and $\delta x_{2}$ respectively. Here $x_{1}=\epsilon_{\theta, \mathrm{c}}^{*}$ and $x_{2}=\epsilon_{\mathrm{eq}, \mathrm{th}}^{*}$.

$(\delta P)_{K}=$ column vector of $K$ parameters, $(K=11)$.

Table 3

Examples of SCODE auxiliary variables. These entities have been removed from the system of equations through direct elimination in favor of the basic variables and parameters

\begin{tabular}{lll}
\hline Variables & Units & Definition \\
\hline$\epsilon_{\theta, \mathrm{c}}$ & $(-)$ & Clad circumferential creep strain \\
$\epsilon_{\text {eq, th }}$ & $(-)$ & Clad equivalent thermal creep strain \\
$G_{\text {pro }}$ & $($ mole & Total fission gas produced in fuel \\
$G_{\text {ret }}$ & $($ mole $)$ & Total fission gas retained in fuel \\
$C_{\mathrm{xe}}$ & $(-)$ & Mole fraction of xenon released from the fuel \\
$\mathrm{QS}$ & $(\mathrm{J} / \mathrm{m})$ & Linear stored heat \\
$\mathrm{I}_{2}$ & $\left(\mathrm{~kg} / \mathrm{m}^{2}\right)$ & Iodine concentration on the clad \\
$\mathrm{DS}$ & $(-)$ & Cumulative clad damage function \\
$\mathrm{PB}$ & $(-)$ & Probability of clad failure by iodine stress corrosion cracking \\
\hline
\end{tabular}


The sets of equations for the $N$ variables take the form of eq. (1). Taking the variations of these equations leads to 33 equations represented below in matrix form.

$$
\left(\delta X^{\prime}\right)_{N}=[A]_{N, N}(\delta X)_{N}+[C]_{N, K}(\delta P)_{K} .
$$

Here $[A]_{N . N}$ is the matrix consisting of the coefficients of the variations in dependent variables (i.e., $\partial f / \partial \boldsymbol{x}$. $\partial \boldsymbol{f} / \partial \boldsymbol{y}, \partial \boldsymbol{g} / \partial \boldsymbol{x}$ and $\partial \boldsymbol{g} / \partial \boldsymbol{y})$ and $[C]_{N, K}$ is the matrix consisting of the coefficients of the variations in parameters of the $K$ equations (i.e., $\partial \boldsymbol{f} / \partial \boldsymbol{p}$ and $\partial \boldsymbol{g} / \partial \boldsymbol{p}$ ). In general, both $[A]_{N, N}$ and $[C]_{N, K}$ will have implicit time dependence through their dependence on the dynamical variables $\boldsymbol{x}$ and $\boldsymbol{y}$.

As a simple example to illustrate our use of notation, consider the following linear system of three equations in three unknowns. The sensitivities are being assessed with respect to the two parameters $p_{1}$ and $p_{2}$.

$$
\dot{x}=B x+p_{1} D y_{1}, \quad y_{1}=p_{2} F y_{2}+G x, \quad y_{2}=p_{1} H y_{1},
$$

then

$$
\delta \dot{x}=B \delta x+p_{1} D \delta y_{1}+D y_{1} \delta p_{1}, \quad \delta y_{1}=p_{2} F \delta y_{2}+F y_{2} \delta p_{2}+G \delta x, \quad \delta y_{2}=p_{1} H \delta y_{1}+H y_{1} \delta p_{1},
$$

and

$$
\left(\boldsymbol{\delta} X^{\prime}\right)_{3}=\left(\begin{array}{l}
\boldsymbol{\delta} \dot{x} \\
\boldsymbol{\delta} y_{1} \\
\boldsymbol{\delta} y_{2}
\end{array}\right)=\left[\begin{array}{lll}
B & p_{1} D & 0 \\
G & 0 & p_{2} F \\
0 & p_{1} H & 0
\end{array}\right]\left(\begin{array}{l}
\boldsymbol{\delta} x \\
\boldsymbol{\delta} y_{1} \\
\boldsymbol{\delta} y_{2}
\end{array}\right)+\left[\begin{array}{ll}
D y_{1} & 0 \\
0 & F y_{2} \\
H y_{1} & 0
\end{array}\right]\left(\begin{array}{l}
\delta p_{1} \\
\delta p_{2}
\end{array}\right),
$$

where $\left(\delta X^{\prime}\right)_{3}=[A]_{3,3}(\delta X)_{3}+[C]_{3,2}(\delta p)_{2}$ is analogous to eq. (14). Note that $[C]_{3,2}$ has time dependence induced through the time dependence of $y_{1}$ and $y_{2}$. At any point in time, however, $[C]_{3,2}$ may be evaluated by using the $y_{1}$ and $y_{2}$ at that operating point. To construct the $[C]_{N, K}$ or $[A]_{N, N}$ matrices only requires evaluation of the appropriate differential forms at the variable values for the current operating point.

The adjoint relations may now be easily set up, with adjoint row vectors defined by the transposed vectors

$$
\begin{aligned}
& (\Phi)_{N}^{\mathrm{T}} \equiv\left(\phi_{1}, \phi_{2}, \psi_{1}, \ldots, \psi_{N-2}\right), \\
& \left(\Phi^{\prime}\right)_{N}^{\mathrm{T}} \equiv\left(-\dot{\phi}_{1},-\dot{\phi}_{2}, \psi_{1}, \ldots, \psi_{N-2}\right) .
\end{aligned}
$$

The adjoint eq. (6) takes the form (since the differential operators act only on $f$ and $g$ but not on $\phi$ or $\psi$ ).

$$
\left(\Phi^{\prime}\right)_{N}^{\mathrm{T}}=(\Phi)_{N}^{\mathrm{T}}[A]_{N, N},
$$

or when transposed

$$
\left(\Phi^{\prime}\right)_{N}=[A]_{N, N}^{\mathrm{T}}(\Phi)_{N},
$$

where $\left(\Phi^{\prime}\right)_{N}$ and $(\Phi)_{N}$ are now column vectors and $[A]_{N, N}^{\mathrm{T}}$ is the transpose of $[A]_{N, N}$. We are now ready to begin the solution procedure for sensitivities.

\section{Solution procedure}

Each time step, SCODE must update the adjoint functions used in calculating the sensitivities. In general, it is necessary to compute a complete set of linearly independent adjoint functions. There will be as many independent sets as there are differential equations in the original system of equations. The adjoint column vectors all evolve according to the same dynamical equation, i.e. eq. (21), and differ only in the choice of initial conditions. In our system two sets of adjoint functions are required and the adjoint 
equation is expressed as

$$
\left[\begin{array}{cc}
-\dot{\boldsymbol{\phi}}_{1,1} & -\dot{\boldsymbol{\phi}}_{1,2} \\
-\dot{\boldsymbol{\phi}}_{2,1} & -\dot{\boldsymbol{\phi}}_{2,2} \\
\psi_{1,1} & \psi_{1,2} \\
\vdots & \vdots \\
\psi_{31,1} & \psi_{31,2}
\end{array}\right]=[A]_{N, N}^{\mathrm{T}}\left[\begin{array}{ll}
\phi_{1,1} & \phi_{1,2} \\
\boldsymbol{\phi}_{2,1} & \phi_{2,2} \\
\psi_{1,1} & \psi_{1,2} \\
\vdots & \vdots \\
\psi_{31,1} & \psi_{31,2}
\end{array}\right]
$$

where now, $\left[\Phi^{\prime}\right]_{N, 2}$ and $[\Phi]_{N, 2}$ are $N \times 2$ matrices since there are two differential equations in our set of equations.

We may, without loss of generality, set the following initial conditions on the adjoint functions

$$
\left\{\begin{array}{l}
\phi_{1,1}(0)=1 \\
\phi_{2,1}(0)=0
\end{array}\right\} \text { and }\left\{\begin{array}{l}
\phi_{1,2}(0)=0 \\
\phi_{2,2}(0)=1
\end{array}\right\},
$$

This gives for eq. (22)

$$
\left[\begin{array}{cc}
-\dot{\phi}_{1,1} & -\dot{\phi}_{1,2} \\
-\dot{\phi}_{2,1} & -\dot{\phi}_{2,2} \\
\psi_{1,1} & \psi_{1,2} \\
\vdots & \vdots \\
\psi_{31,1} & \psi_{31,2}
\end{array}\right]=[A]_{N, N}^{\mathrm{T}}\left[\begin{array}{ll}
1 & 0 \\
0 & 1 \\
\psi_{1,1} & \psi_{1,2} \\
\vdots & \vdots \\
\psi_{31,1} & \psi_{31,2}
\end{array}\right],
$$

We must now solve eq. (24) for $[\psi]_{N-2,2}$ to obtain the remaining initial conditions for the $\psi$-type adjoint functions from the constraint equations. To do this, we rewrite eq. (24) by decomposing the matrix multiplication into the part multiplying the $2 \times 2$ matrix and the part multiplying the $\psi$ vectors.

$$
[\Phi]_{3: N, 2} \equiv[A]_{3: N, 1: 2}^{\mathrm{T}}+[A]_{3: N, 3: N}^{\mathrm{T}}[\psi]_{N-2,2},
$$

where $[\psi]_{N-2,2}$ is the matrix of the two column vectors $\left(\psi_{1, i}, \psi_{2, i}, \ldots, \psi_{31, i}\right)^{\mathrm{T}}, i=1,2$ and $(A)_{3: N, 1: 2}^{\mathrm{T}}$ are the first and second columns of the $[A]_{N, N}^{\mathrm{T}}$ matrix including elements form the 3 rd to the $N$ th row. Likewise, $[A]_{3: N, 3: N}^{\mathrm{T}}$ is that part of the $[A]_{N, N}^{\mathrm{T}}$ matrix restricted to elements from the 3 rd to the $N$ th rows and columns.

Eq. (25) admits a direct matrix solution for the $\psi$ matrix

$$
[\psi]_{N-2,2}=\left[1-[A]_{3: N, 3: N}^{\mathrm{T}}\right]^{-1}[A]_{3: N, 1: 2}^{\mathrm{T}},
$$

where 1 is the unity matrix and the -1 denotes matrix inversion. Between eqs. (23) and (26) we have the full set of $2 N$ initial conditions on the $2 N$ adjoint function of $[\Phi]_{N, 2}$.

At the end of each time step we must update the adjoint functions to track their time evolution. Hence we would like a transformation $B$ that does the following

$$
[\Phi(t+\Delta t)]_{N, 2}=[B]_{N, N}[\Phi(t)]_{N, 2} \text {, }
$$

where the elements of $[\Phi]_{N, 2}$ are known from the previous time step. Taking the numerical approximation for derivatives and midpoints

$$
\dot{\phi}(t+\Delta t / 2)=\frac{\phi(t+\Delta t)-\phi(t)}{\Delta t}, \quad \psi(t+\Delta t / 2)=\frac{\psi(t+\Delta t)+\psi(t)}{2}
$$


and using eq. (22) in a forward integration mode, we find

$$
[B]_{N, N}=\left[\begin{array}{lllll}
\left(-\Delta t A_{1,1}+1\right) & -\Delta t A_{1,2} & -\Delta t A_{1,3} & \cdots & -\Delta t A_{1, N} \\
-\Delta t A_{2,1} & \left(-\Delta t A_{2,2}+1\right) & -\Delta t A_{2,3} & \cdots & -\Delta t A_{2, N} \\
2 A_{3,1} & 2 A_{3,2} & \left(2 A_{3,3}-1\right) & \cdots & 2 A_{3, N} \\
\vdots & \vdots & \vdots & \cdots & \vdots \\
2 A_{N, 1} & \vdots & \cdot & \cdots & \left(2 A_{N, N}-1\right)
\end{array}\right] .
$$

The variational relation, eq. (13), can now be used to obtain the sensitivities for $x_{1}$ and $x_{2}$. The equation takes the form

$$
\left[\begin{array}{ll}
\phi_{1,1}(t) & \phi_{1,2}(t) \\
\phi_{2,1}(t) & \phi_{2,2}(t)
\end{array}\right]\left(\begin{array}{l}
\delta x_{1} \\
\delta x_{2}
\end{array}\right)=\int_{0}^{t}[\Phi]_{2, N}^{\mathrm{T}}[C]_{N, K}(\delta P)_{K} \mathrm{~d} t \equiv\left[C^{\prime}\right]_{2, K}(\delta P)_{K} .
$$

The $C$-prime matrix is a time integral and may be written in a recursive form used to perform its value updating,

$$
\left[C^{\prime}(t+\Delta t)\right]_{2, K}=\left[C^{\prime}(t)\right]_{2, K}+\Delta t[\Phi(t)]_{2, N}^{\mathrm{T}}[C(t)]_{N, K^{*}}
$$

Eq. (30) can be inverted to obtain

$$
\left(\begin{array}{l}
\delta x_{1} \\
\delta x_{2}
\end{array}\right)=\left[\begin{array}{ll}
\phi_{1,1}(t) & \phi_{1,2}(t) \\
\phi_{2,1}(t) & \phi_{2,2}(t)
\end{array}\right]^{-1}\left[C^{\prime}\right]_{2, K}(\delta P)_{K},
$$

or

$$
(\delta X)_{1: 2}=[G]_{2, K}(\delta P)_{K},
$$

where

$$
[G]_{2, K}=\left[\begin{array}{ll}
\phi_{1,1}(t) & \phi_{1,2}(t) \\
\phi_{2,1}(t) & \phi_{2,2}(t)
\end{array}\right]^{-1}\left[C^{\prime}\right]_{2, K} .
$$

The $G$ matrix gives the $x_{1}$ and $x_{2}$ sensitivities, that is,

$$
[\delta X / \delta P]_{1: 2, K}=[G]_{2, K}
$$

The other unknowns $(\delta X)_{3: N} \equiv\left(\delta X^{\prime}\right)_{3: N}$ can now be solved by using the $3: N$ components from eq. (14). Noting $\delta X^{\prime}$ and $\delta X$ are identical for these components eq. (14) reduces to

$$
\left[1-[A]_{3: N, 3: N}\right](\delta X)_{3: N}=[A]_{3: N, 1: 2}(\delta X)_{1: 2}+[C]_{3: N, K}(\delta P)_{N} .
$$

Substituting for $\delta X_{1: 2}$ from eq. (33) gives

$$
\left[1-[A]_{3: N, 3: N}\right](\delta X)_{3: N}=\left[[A]_{3: N, 1: 2}[G]_{2, K}+[C]_{3: N, K}\right](\delta P)_{K} .
$$

Finally, we have for the remaining sensitivities

$$
[\delta X / \delta P]_{3: N, K}=\left[1-[A]_{3: N, 3: N}\right]^{-1}\left[[A]_{3: N, 1: 2}[G]_{2, K}+[C]_{N, K}\right]
$$

To aid in physical interpretation, sensitivity results have been cast in the form of significance. That is, if

$$
\partial X_{i} / \partial P_{j}=\text { sensitivity of output } X_{i} \text { to input } P_{j},
$$

then the significance associated with that sensitivity is defined as

$$
S_{i j}=\frac{P_{j}}{X_{i}} \frac{\partial X_{i}}{\partial P_{j}}
$$


The units of significance are percent change of output per percent change of input. The sensitivities of $X_{1}, X_{2}, \ldots, X_{33}$ with respect to $P_{1}, P_{2}, P_{3}, \ldots, P_{11}$ (thus resulting in $33 \times 11$ sensitivities) have thus been reduced to a problem in matrix algebra, solved by SCODE to obtain FCODE-ALPHA sensitivities.

\section{Results and discussion}

Tables 4 and 5 are sample SCODE output matrices containing over 360 significances each with variables and parameters defined in tables 1 and 2 . This particular analysis was performed on the FCODE-ALPHA output from a rod that was operated for two time steps, representative of start-of-life conditions. For table 4 the last power step was held at $9200 \mathrm{~W} / \mathrm{m}$ to ensure open gap conditions. Table 5 portrays a closed gap condition, the second power step being set at $29500 \mathrm{~W} / \mathrm{m}$ to achieve this. Significances are read off the matrix as a percent change in the column (basic) variable given a percent change in the row variable (parameter). For example, the figure in the $(1,1)$ position of the table 4 matrix is interpreted as $\delta \epsilon_{\theta, \mathrm{c}}^{*} / \delta t_{\mathrm{F}}=$ $(0.65)\left(\epsilon_{\theta, \mathrm{c}}^{*} / t_{\mathrm{F}}\right)$. Care must be exercised in interpretation since in a case such as this the sensitivity value will have opposite sign to the significance value owing to the negativity of $\epsilon_{\theta, c}^{*}$.

Since the data is so voluminous only selected results will be discussed. Emphasis is placed on the significances which are largest. For the open gap case of table 4, the following observations are noteworthy:

(a) A heavy dependence of creepdown (No. 1-2) on $R_{\text {Cl.fab }}$ and to a lesser extent on $R_{\text {Co,fab }}$. The large negative sensitivity (but positive significance) $\delta \epsilon_{\theta, \mathrm{c}}^{*} / \delta R_{\mathrm{Cl}, \mathrm{fab}}$ is attributable to the reduction in clad wall thickness and elevated clad temperatures (due to larger fuel/clad gap) that result from positive $R_{\mathrm{CI}, \mathrm{fab}}$ variation.

(b) The fuel/clad gap (No. 28) is highly sensitive to $R_{\mathrm{F}, \text { fab }}$ but with complementary signs. The magnitude of these significances demonstrates the obvious fact that a small relative change in fuel or inner clad dimensions produces a large relative change in the gap, the amplification factor being roughly $R_{\mathrm{CI}, \mathrm{fab}} /\left(R_{\mathrm{CI}, \mathrm{fab}}-R_{\mathrm{F}, \mathrm{fab}}\right)$.

(c) The fabricated dimension $R_{F, f a b}$ has a large influence on variables such as gap conductance (No. 21), fuel cracking (No. 16) and fuel temperatures (No. 20 and No. 23), which is easily understood in terms of the reduction of temperature drop across the gap with decreased gap size. In these respects $R_{\text {CI,fab }}$ plays a complementary role.

(d) The parameters $R_{\text {CI,fab }}$ and $R_{\text {Co,fab }}$ have appreciable but complementary effects on cladding stresses (No. 3-5), which are understandable since these stresses are proportional to the clad radius to wall thickness ratio.

(e) Outer clad pressure, $p_{\mathrm{O}}$, has a strong effect on creepdown, the significance value being 6.0 . The magnitude of this effect derives from the high power with which stress enters the (strain hardening) creep law.

(f) The current linear power, $Q_{\mathrm{L}}$, and linear power scale factor $\alpha_{\mathrm{O}}$, enter predominantly through their effects on the surface crack width (No. 16). This is tracable to the steep dependence (roughly cubic) of crack width on fuel temperature drop. Note that the sensitivity of centerline temperature (No. 23) to $Q_{\mathrm{L}}$ is 0.59 and fuel surface temperature (No. 20) is only 0.27 . The departure of these values from unity illustrates the strong countervailing influence of the temperature drop across the gap in opposing thermal expansion.

(g) The parameters bu den and $P_{\mathrm{fab}}$ effect only one variable significantly, the swelling strain (No. 23). Swelling decreases when the characteristic burnup for densification is increased, as expected. Likewise an initial increase in fabricated porosity allows for greater fractional fuel swelling.

(h) The step time, $t_{\mathrm{F}}$, enters mainly through its influence on creepdown and gas release.

(i) The clad outer temperature, $T_{\mathrm{CO}}$, has an appreciable positive effect on relative creepdown and affects fuel temperatures with a positive, but less than unity, significance. The departure from unity is understandable for the same reason given in describing the $Q_{\mathrm{L}}$ sensitivities. 


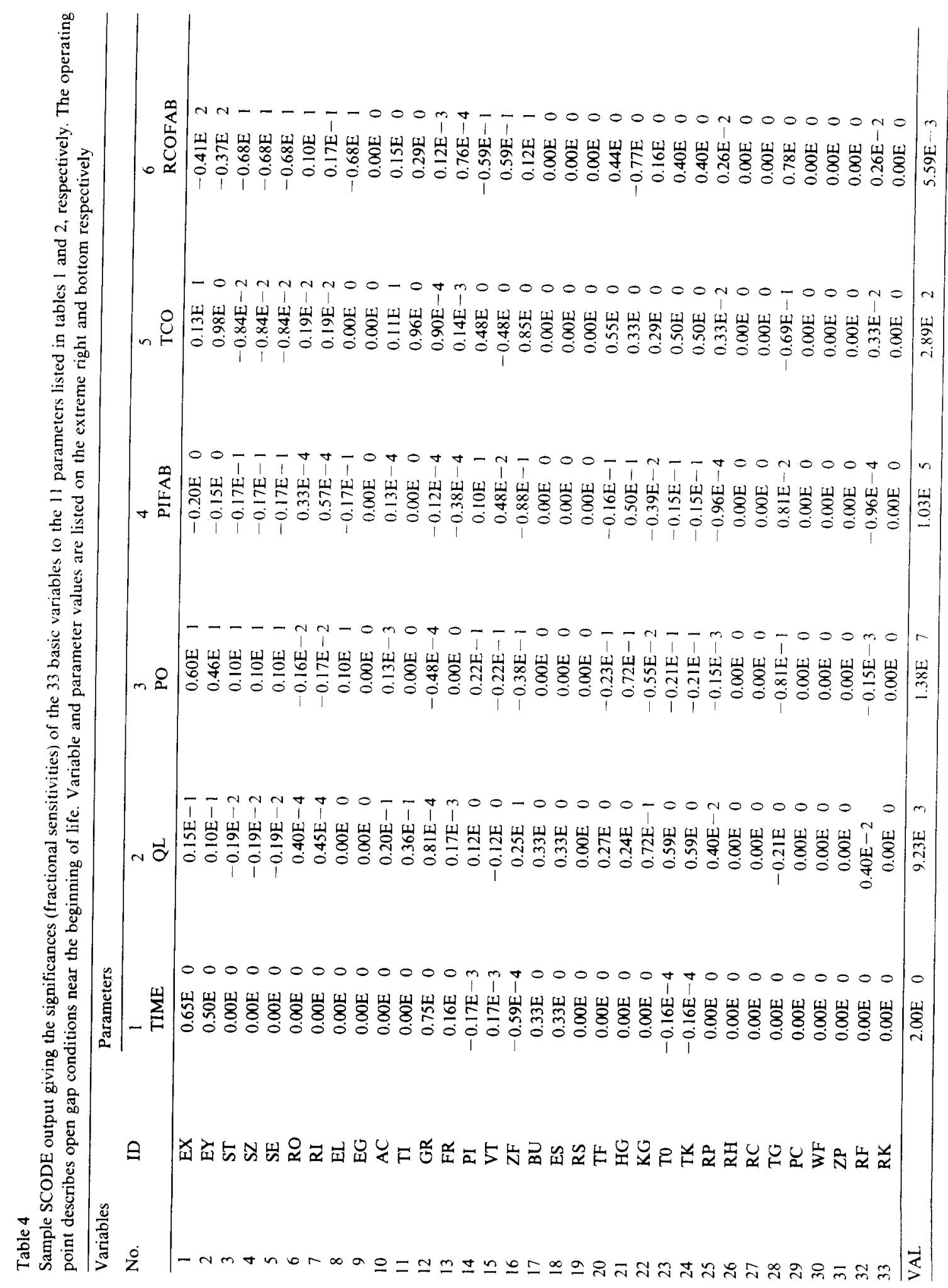




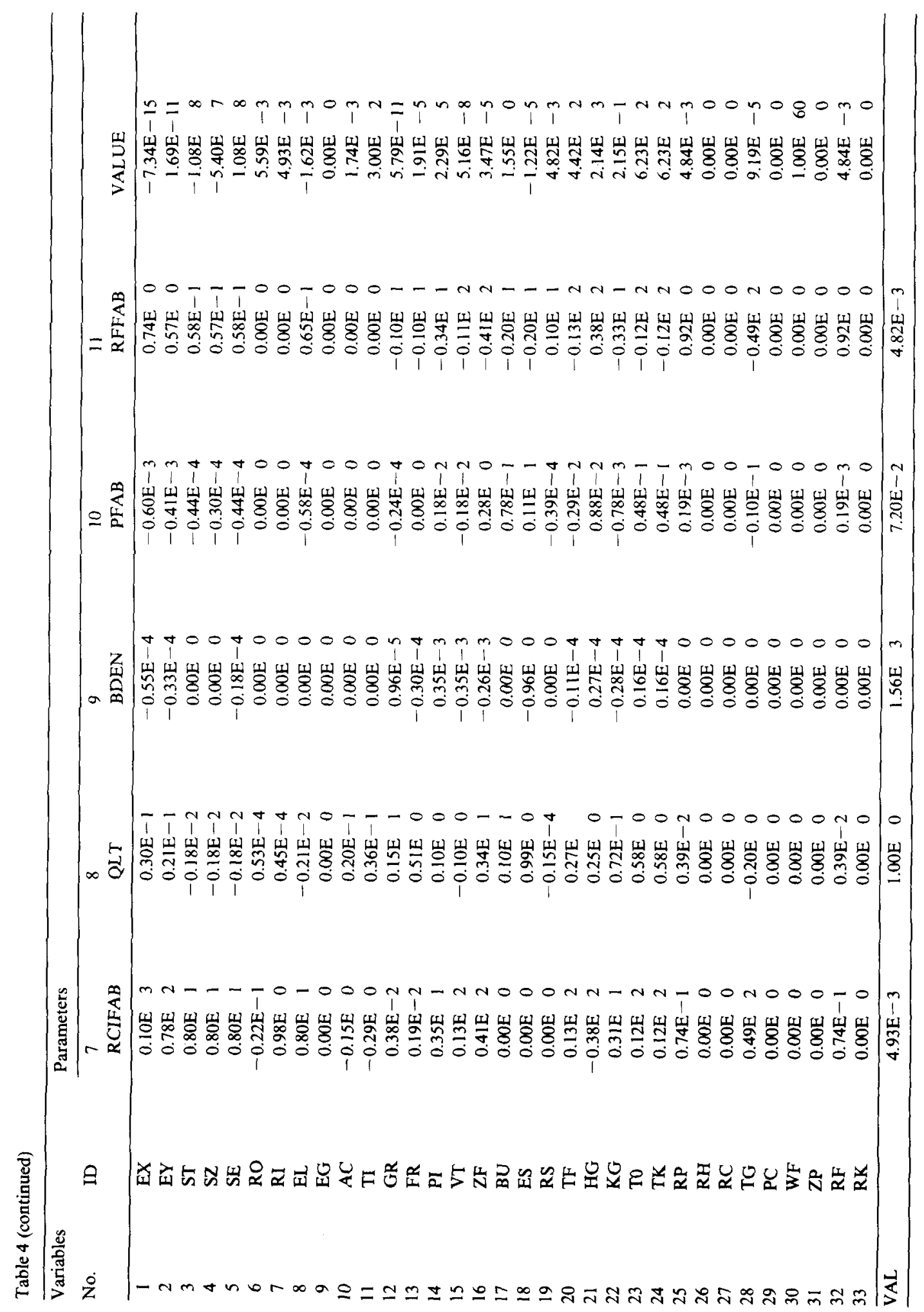




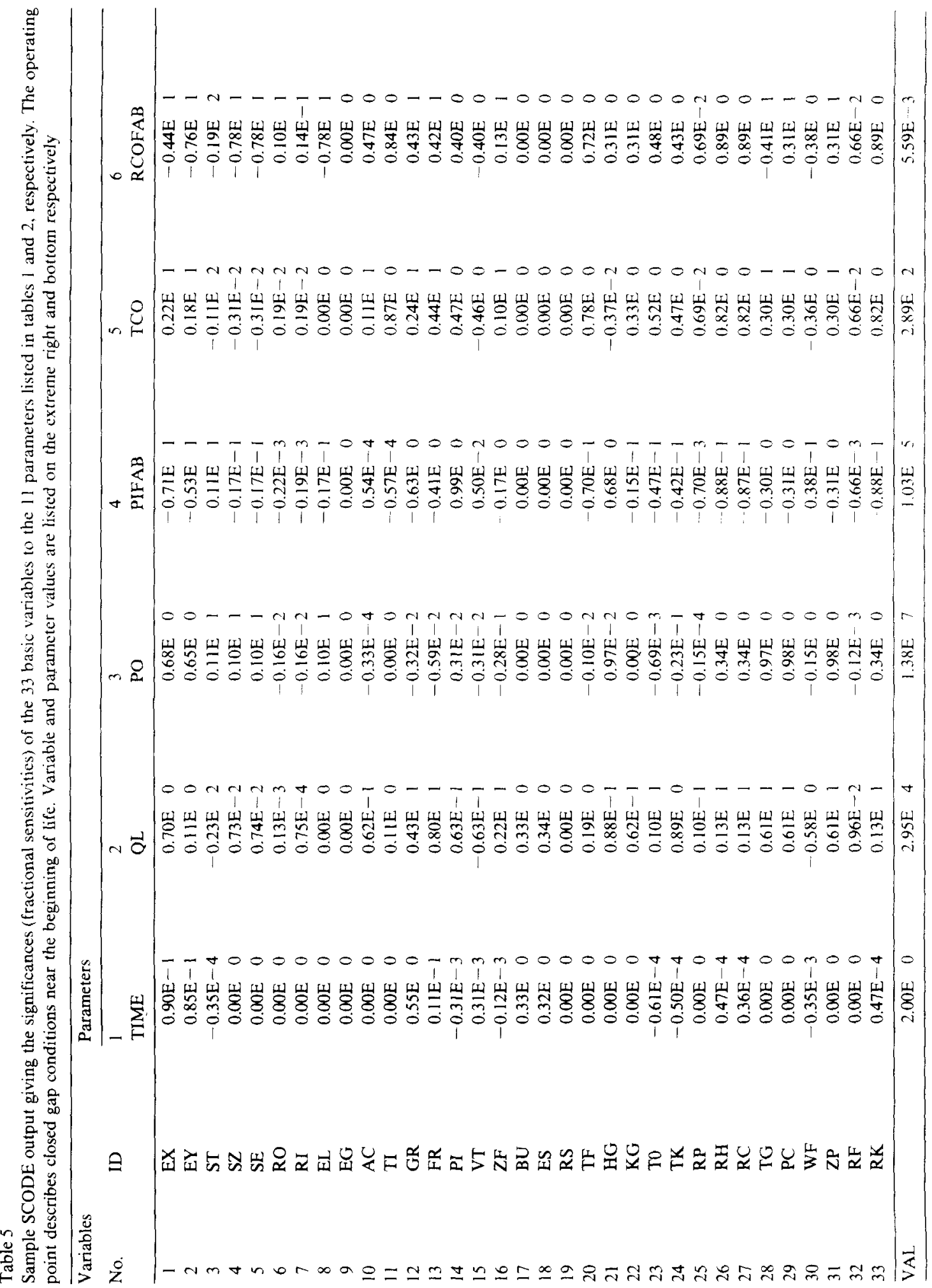




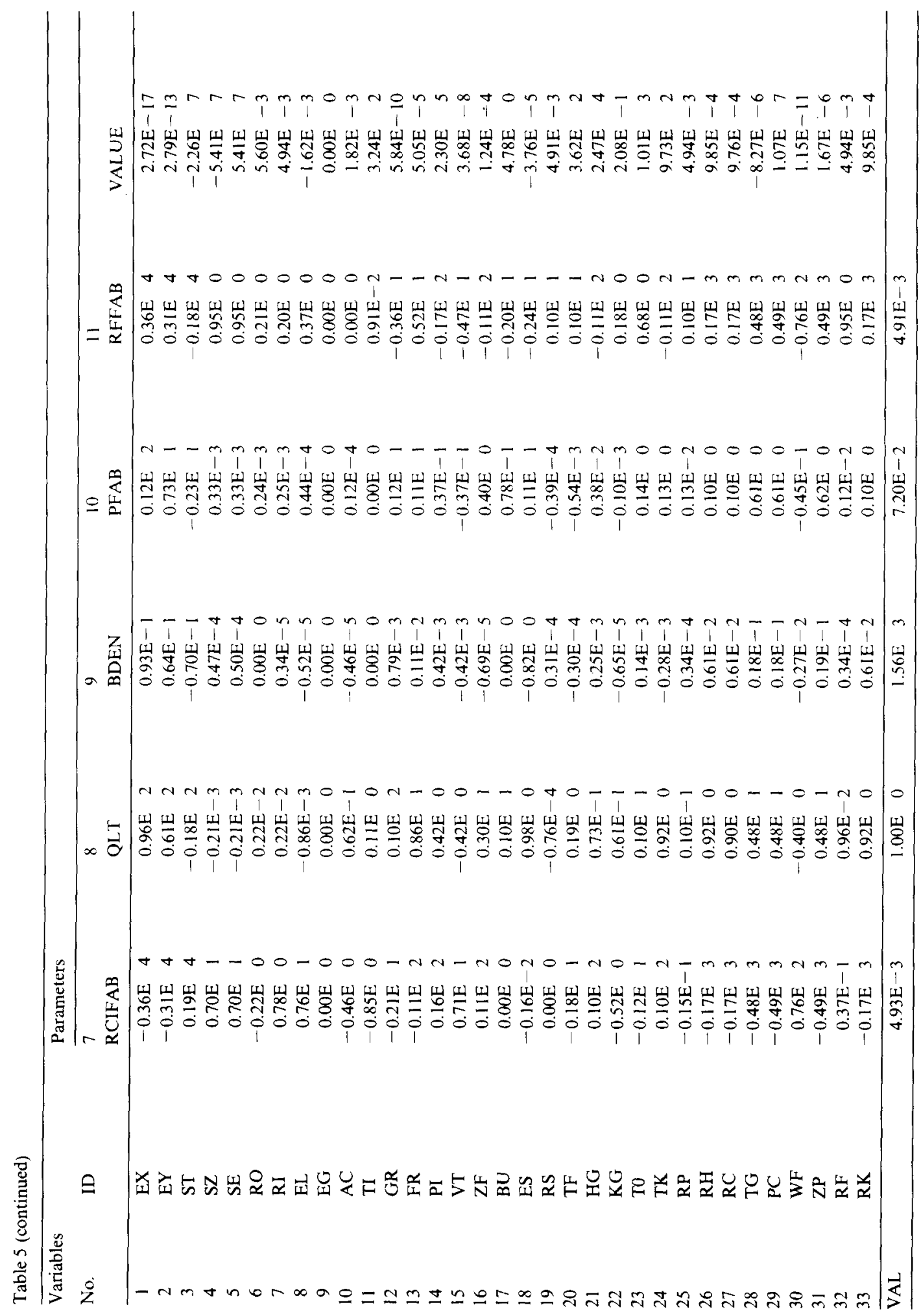


A rather different scenario is given in table 5, which presents sensitivity information under closed gap conditions. We focus our attention on the major differences between the open and closed gap cases.

(a) The largest significance value is still for $\delta \epsilon_{\theta, c}^{*} / \delta R_{\text {Cl,fab }}$, but under closed gap conditions it is some 35 times larger and of opposite sign. This is attributable to the change in sign and magnitude of $\epsilon_{\theta, c}^{*}$ itself. The fact that $\delta \epsilon^{*}{ }_{\theta, \mathrm{c}} / \delta R_{\mathrm{F}, \text { fab }}$ is of equal magnitude and complementary sign is indicative that the major determinant of creep has shifted from gas outer pressure to contact pressure (unrestrained fuel/clad gap overlap).

(b) Sensitivities to fabricated fuel and inner clad dimensions are even larger than seen in the open gap case. Circumferential stress (No. 3) is significant at nearly the 2000 level; contact pressure (No. 29) and fuel/clad gap (No. 28) have significances of nearly 500; and fractional gas release (No. 13), inner gas pressure (No. 14), fuel surface crack width (No. 16), gap conductance (No. 21), temperature at the cracking radius (No. 24) and restrained cracking radius (No. 33) all have large significances.

(c) In contrast to the open gap case, under closed gap conditions increasing $R_{\text {F.fab }}$ produces a marked decrease in gap conductance (No. 21) and a corresponding increase in fuel temperatures (No. 20 and 23). This result could not have been foreseen and is even stranger in view of the increased gap conductivity (No. 22). The resolution of this seeming paradox lies in the strong role that the internal gas pressure (No. 14) exerts on gap conductance under closed gap conditions. Reduced gas pressure serves to decrease the gap conductance. The overall gas release (No. 12) decreases with increasing $R_{\mathrm{F} \text {,fab }}$, this being responsible for the lower gas pressure. Curiously, the release fraction on the current time step (No. 13) is increased, but apparently not enough to offset its first-time-step decrease. This example highlights the often complex and occasionally counter-intuitive way in which variables can interact, not only on the current time step, but also over the entire history since startup.

(d) The role of linear power, $Q_{\mathrm{L}}$, is more important under closed gap conditions. High $Q_{\mathrm{L}}$ sensitivities, which did not exist for open gap, are seen for gas release (No. 12 and 13), contact pressure (No. 29), various fuel cracking model variables and particularly circumferential stress (No. 3). The seemingly paradoxical low sensitivity of equivalent stress (No.5) occurs because, in the Tresca formulation, equivalent stress is independent of circumferential stress whenever its value lies between that of the axial and radial stress values. This situation, if fact, exists since the (tensile) contact pressure is not quite large enough to overcome the (compressive) outer gas pressure.

(e) The situation for $T_{\mathrm{CO}}$ is similar to that of the parameter $Q_{\mathrm{L}}$, with the same variables experiencing heightened sensitivity under closed gap conditions.

(f) The high significances seen for the creep variables are somewhat artificial in that they appear amplified because of the relatively small value of the creep variables themselves.

In general, the greatest sensitivities in the model stem from the geometric parameters $R_{\mathrm{CI}, \mathrm{fab}}, R_{\mathrm{F}, \mathrm{fab}}$ and

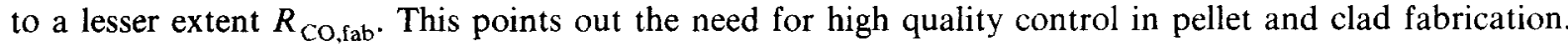
From the fuel modeling point of view, such effects as cladding ovality and eccentric positioning of the fuel within the clad merit close scrutiny. Of course, mechanisms that directly effect the gap, such as fuel relocation and fission gas bubble swelling, are clearly important in fuel performance modeling.

The value of sensitivities lies in the capability of uncovering dependencies between large numbers of variables that are impossible for the human mind to trace. The reader should be cautioned in applying these results to obtain quantitative answers to macroscopic changes in a system. By the definition of a sensitivity $\partial X / \partial p$, it is the infinitesimal change in $X$ given an infinitesimal change in $p$. In the case of a simple function of one parameter, $X=X(p)$, the sensitivity is the derivative and is valid only for infinitesimal distances about the operating point. This means that the values of table 4 give the direction (sign) and the magnitude of the variation at the operating point. Extrapolations to the macroscopic, such as expecting that a $50 \%$ change in $p$ will produce a $100 \%$ change in $X$ (if $\partial X / \partial p=2.0$ ) are not automaticall; valid. 


\section{Summary}

Adjoint sensitivity analysis is performed on results of the fuel behavior code FCODE-ALPHA, which describes the thermomechanical behavior of a fuel rod subject to a specific set of input conditions. The adjoint sensitivity analysis is performed by SCODE in 31 constraint and 2 differential equations for their variations with respect to 11 input parameters. The result is 363 significances. Since the solution procedure entails solving 33 equations simultaneously, the magnitude and sign of complicated dependencies is determined which is otherwise beyond the scope of comprehension. Results reveal some interesting inter-relationships between variables. Sensitivities should be used in their proper context, that is on a microscopic basis, and not for gross macroscopic extrapolations as this violates the premise of the variation.

\section{Acknowledgment}

The work was supported by the Electric Power Research Institute under contract RP971-2.

\section{References}

[1] R.A. Christensen, SPEAR fuel reliability code system: general description, Electric Power Research Institute, Interim Report EPRI NP-1378 (March 1980).

[2] L.W. Negal and R.A. Rohrer, Computer analysis of non-linear circuits excluding radiation (CANCER), IEEE J. Solid-State Circuits SC-6 (1971) 166-182.

[3] S.W. Director and R.A. Rohrer, The generalized adjoint network and network sensitivities, IEEE Trans. Circuit Theory CT-16 (1969) 318-323.

[4] J. Vagners, Optimization techniques, in: Carl E. Pearson, ed., Handbook of Applied Mathematics (Van Nostrand, New York. 1974) pp. 1104-1180.

[5] R.A. Christensen, Fuel rod mechanical performance modeling; Task 3: fuel rod modeling and decision analysis, Electric Power Research Institute, Eighth Quarterly Progress Report, FRMPM31-1 (May 1979) pp. 5-1-5-59.

[6] R.F. Eilbert, Sensitivities in F-CODE, Chapt. 3, in: R. Christensen, Fuel Rod Mechanical Performance Modeling; Task-3; Fuel Rod Modeling and Decision Analysis, FRMPM16-1, Electric Power Research Institute RP971-2, Third Quarterly Progress Report (Feb. 1978) pp. 93-138.

[7] G.S. Was, R.A. Christensen and R.F. Eilbert, Sensitivities relations developments, Chapt. 5, in: R. Christensen, Fuel Rod Mechanical Performance Modeling; Task-3: Fuel Rod Modeling and Decision Analysis, FRMPM30-1, Electric Power Research Institute RP971-2, Sixth Quarterly Progress Report (Feb. 1979) pp. 5-1-5-50.

[8] D.R. Harris, Sensitivity of nuclear fuel cycle costs to uncertainties in nuclear data and methods, Doctoral Thesis, Rensselaer Polytechnic Institute (1976).

[9] D.R. Harris and M. Becker, Nonlinear perturbation technique for the nuclear fuel cycle, Trans. Amer. Nucl. Soc. 23 (1976) 534.

[10] J.M. Ryskamp, M. Becker and D.R. Harris, Sensitivity of LWR fuel cycle costs to uncertainties in detailed thermal cross sections, Proc. Topical Meeting: Sensitivity and Uncertainty Analysis, Oak Ridge National Laboratory, Aug. 22-24, 1978 